\title{
Active Expiration Induced by Excitation of Ventral Medulla in Adult Anesthetized Rats
}

\author{
Silvia Pagliardini, ${ }^{1}$ Wiktor A. Janczewski, ${ }^{1}$ Wenbin Tan, ${ }^{1}$ Clayton T. Dickson, ${ }^{2,3,4}$ Karl Deisseroth,,${ }^{5,6}$ and \\ Jack L. Feldman ${ }^{1}$ \\ ${ }^{1}$ Systems Neurobiology Laboratory, Department of Neurobiology, David Geffen School of Medicine, University of California, Los Angeles, Los Angeles, \\ California 90095-1763, Departments of ${ }^{2}$ Psychology, ${ }^{3}$ Physiology, and ${ }^{4}$ Centre for Neuroscience, University of Alberta, Edmonton, Alberta T6G 2E9, Canada, \\ and Departments of ${ }^{5}$ Bioengineering and ${ }^{6}$ Psychiatry, Stanford University, Stanford, California 94305
}

Data from perinatal and juvenile rodents support our hypothesis that the preBötzinger complex generates inspiratory rhythm and the retrotrapezoid nucleus-parafacial respiratory group (RTN/pFRG) generates active expiration (AE). Although the role of the RTN/pFRG in adulthood is disputed, we hypothesized that its rhythmogenicity persists but is typically silenced by synaptic inhibition. We show in adult anesthetized rats that local pharmacological disinhibition or optogenetic excitation of the RTN/pFRG can generate AE and transforms previously silent RTN/pFRG neurons into rhythmically active cells whose firing is correlated with late-phase active expiration. Brief excitatory stimuli also reset the respiratory rhythm, indicating strong coupling of AE to inspiration. The AE network location in adult rats overlaps with the perinatal pFRG and appears lateral to the chemosensitive region of adult RTN. We suggest that (1) the RTN/pFRG contains a conditional oscillator that generates AE, and (2) at rest and in anesthesia, synaptic inhibition of RTN/pFRG suppresses AE.

\section{Introduction}

The preBötzinger complex (preBötC) in the ventrolateral medulla (Smith et al., 1991; Gray et al., 1999, 2001) plays a key role in respiratory rhythmogenesis (Feldman and Del Negro, 2006; Thoby-Brisson et al., 2009). A region just rostral to the preBötC, the retrotrapezoid nucleus/parafacial respiratory group (Onimaru and Homma, 2003) (RTN/pFRG), may also play a critical role in ontogeny (Thoby-Brisson et al., 2009) and generation (Feldman and Smith, 1989; Onimaru and Homma, 2003) of respiratory rhythm, as well as in chemoreception (Smith et al., 1989; Mulkey et al., 2004). We postulated (Janczewski and Feldman, 2006) that these two regions are coordinated, with preBötC generating inspiration, and RTN/pFRG generating enhanced expiratory airflow [i.e., active expiration (AE)]. When both inspiration and expiration are active, we proposed that both oscillators are rhythmically engaged and are strongly coupled (Janczewski and Feldman, 2006).

The RTN/pFRG is a heterogeneous group of neurons surrounding the facial nucleus (VIIn) with two functional neuronal populations that may overlap, presumptive rhythmogenic pFRG neurons and chemosensitive RTN neurons. In vitro studies in

Received Oct. 5, 2010; revised Dec. 2, 2010; accepted Dec. 21, 2010.

This work was supported by National Institutes of Health Grants R01 HL-70029 and R21 HL-94811. Canadian Institute of Health Research and Alberta Heritage Foundation for Medical Research (AHFMR) postdoctoral fellowships funded S.P. C.T.D. is an AHFMR senior scholar.

Correspondence should be addressed to Jack L. Feldman, Systems Neurobiology Laboratory, Department of Neurobiology, David Geffen School of Medicine, University of California, Los Angeles, Box 951763, Los Angeles, California 90095-1763. E-mail: feldman@ucla.edu.

S. Pagliardini's present address: Department of Physiology, Centre for Neuroscience, 5005 Katz Building, University of Alberta, Edmonton, Alberta T6G 2E1, Canada.

DOI:10.1523/JNEUROSCI.5338-10.2011

Copyright $\odot 2011$ the authors $\quad 0270-6474 / 11 / 312895-11 \$ 15.00 / 0$ perinatal rats show rhythmically active respiratory neurons ventrolateral to the VIIn that discharge immediately before inspiration (Janczewski et al., 2002; Onimaru and Homma, 2003; Onimaru et al., 2006). In juvenile rats in vivo, brainstem transections that remove the RTN/pFRG but leave the preBötC intact abolish AE but have limited effect on inspiration (Janczewski and Feldman, 2006). Moreover, in en bloc preparations from neonatal rats and in juvenile rats, opiates can induce quantal slowing [i.e., skipped inspiratory bursts (Mellen et al., 2003; Janczewski and Feldman, 2006)]. We postulated that the RTN/pFRG is a conditional oscillator that initiates and maintains $\mathrm{AE}$ (i.e., the "two-oscillator" hypothesis).

With respect to expiratory activity, adult rats differ from perinatal and juvenile rats. RTN neurons with various respiratory-modulated patterns are present in adults (Pearce et al., 1989; Connelly et al., 1990), although during resting conditions or under anesthesia there is a conspicuous absence of AE (Iizuka and Fregosi, 2007) (but see de Almeida and Kirkwood, 2010; de Almeida et al., 2010) and/or RTN rhythmicity (Mulkey et al., 2004; Fortuna et al., 2008). Rapid suppression of preBötC activity in awake adult rat leads to apnea with no evidence of AE (Tan et al., 2008). Humans typically lack AE when at rest (Iscoe, 1998; Abraham et al., 2002), although expiratory motor activity driving expiratory airflow is turned on during exercise. Based on these developmental differences, we postulated that RTN/pFRG rhythmicity is actively suppressed in anesthetized adult rodents. Here, we explore the possibility that this suppression is attributable to inhibition via GABA and/or glycine neurotransmission.

We tested the two-oscillator hypothesis by activating RTN/ pFRG neurons using either localized disinhibition or by localized excitation mediated by photoactivation of neuronally expressed 
channelrhodopsin (ChR2). We could induce AE in adult anesthetized rats by either method. Stimulation of the lateral part of the RTN/pFRG transformed a resting breathing pattern with no $\mathrm{AE}$ into one with $\mathrm{AE}$, associated with rhythmic late expiratory activity in previously silent/tonic RTN/pFRG neurons. These results are consistent with our hypothesis that $\mathrm{AE}$ can result from activation of a conditional RTN/pFRG oscillator that persists in adulthood and is coupled to the inspiratory rhythm generator. Preliminary results were presented in abstract form (Pagliardini et al., 2009a,b).

\section{Materials and Methods}

Bicuculline/strychnine injection experiments. Animal handling and experimental protocols were approved by the University of California, Los Angeles, Chancellor's Animal Research Committee. Thirty adult male Sprague Dawley rats (300-350 g) were used for acute experiments. Rats were initially anesthetized in halothane ( $3 \%$ in air) while the femoral vein was cannulated and urethane (1.5-1.7 g/ kg body weight) was gradually delivered to induce permanent and irreversible anesthesia. Additional doses of anesthesia were delivered as necessary to maintain the level of anesthesia. The trachea was cannulated and respiratory flow was detected with a flow head connected to a transducer to measure airflow (GM Instruments). Coupled EMG wire electrodes (Cooner Wire) were inserted into the genioglossal (GG), oblique abdominal (ABD), diaphragm (DIA) muscles, and occasionally in the levator labii superioris (LL), which dilates the nostril and elevates the upper lip and is innervated by the buccal branch of the facial nerve. Wires were connected to amplifiers (Grass Model P511; Grass Instruments) and activity was sampled at 2-4 $\mathrm{kHz}$ (Powerlab 16SP; ADInstruments). To eliminate confounding effects induced by stimulation of vagal reflexes, rats were vagotomized by resecting a portion of the vagus nerve $(2 \mathrm{~mm})$ at midcervical level. Body temperature was kept constant at $37 \pm 1^{\circ} \mathrm{C}$ with a servo-controlled heating pad (Fine Science Tools). Eight rats were used for testing the response to $5 \% \mathrm{CO}_{2}$ in the inspired air (5 min stimulation). The remaining rats were then placed in a stereotaxic frame with bregma $5 \mathrm{~mm}$ below lambda. Bicuculline and strychnine (BIC/STRY) $(50 \mu \mathrm{M} ; 200 \mathrm{nl} / \mathrm{side}$; SigmaAldrich) diluted in saline $(n=18)$ or saline $(n=4)$ were pressure injected through a sharp glass electrode ( $40 \mu \mathrm{m}$ tip diameter) into the RTN/pFRG. Coordinates were as follows (in $\mathrm{mm}$ ): 1.8 rostral, 2.5 lateral, and 3.5 ventral to the obex. Additional injections (along the rostrocaudal and mediolateral axes) were performed to identify the extent of the regions responsive to drug application.

In some experiments, fluorescent beads $(0.2 \mu \mathrm{m}$ diameter, $0.2 \%$ solution; Invitrogen) were added to the injected solution to locate the injection site.

Virus preparation and injection into the $p F R G$. The lentivirus containing the mutated channelrhodopsin-2 protein (ChR2-H134R) fused with enhanced yellow fluorescent protein (EYFP) driven by the neuronal specific promoter synapsin (SYN) (SYN-ChR2-EYFP). The ChR2 with H134R mutation when light activated produces a twofold increase in steady-state current compared with the wild-type form of ChR2 (Adamantidis et al., 2007; Gradinaru et al., 2007). The virus was produced according to the detailed protocol described by Zhang et al. (2007) and briefly described below. 293FT cells (Invitrogen) were cotransfected with SYN-ChR2-EYFP lentiviral plasmid and helper vectors (pCMV deltaR8.74 and pMD2.G) using a calcium coprecipitation method. Twentyfour hours after transfection, the cells were treated by $5 \mathrm{~mm}$ sodium butyrate (Sigma-Aldrich) for another $24 \mathrm{~h}$. The supernatant was collected, filtered, and centrifuged through $20 \%$ sucrose cushion at $120,000 \times g$ at $4^{\circ} \mathrm{C}$ for $2 \mathrm{~h}$. The viral pellet was resuspended in sterile PBS to obtain a titer of $10^{10} \mathrm{pfu} / \mathrm{ml}$.

Viral injection into the pFRG. A separate cohort of rats was used for the laser photostimulation experiments. The $300 \mathrm{~g}$ adult Sprague Dawley rats were anesthetized with ketamine $(90 \mathrm{mg} / \mathrm{kg})$ and xylazine $(10 \mathrm{mg} / \mathrm{kg})$ and positioned in a stereotaxic frame with bregma $5 \mathrm{~mm}$ below lambda and either the lentivirus SYN-ChR2-EYFP $\left(0.4-0.5 \mu \mathrm{l} ; 10^{9}-10^{10} \mathrm{pfu} / \mathrm{ml}\right.$; $n=18)$ or SYN-EYFP $\left(0.4-0.5 \mu \mathrm{l} ; 10^{9}-10^{10} \mathrm{pfu} / \mathrm{ml} ; n=6\right)$ were pressure injected either unilaterally or bilaterally through a sharp glass elec- trode (40 $\mu \mathrm{m}$ tip diameter) into the RTN/pFRG. The electrodes were left in place for 5 min after each injection to minimize backflow of virus up the electrode track. Coordinates were as follows (in $\mathrm{mm}$ ): 1.8 rostral, 2.5 lateral, and 3.5 ventral to the obex. Postoperatively, rats received buprenorphine $(0.1 \mathrm{mg} / \mathrm{kg})$ and oral antibiotics in their drinking water for $14 \mathrm{~d}$. Rats recovered for 4 weeks with food and water ad libitum before laser stimulation experiments.

Laser stimulation experimental protocol. Four weeks after surgery, rats were anesthetized with halothane (3\% in air) while the femoral vein was cannulated, and then urethane (1.5-7 g/kg body weight) was gradually delivered to induce permanent and irreversible anesthesia. Respiratory flow was measured via a tracheal cannula and EMG wires were implanted in ABD, GG, DIA, and LL muscles. Rats were vagotomized and then positioned supine in a stereotaxic frame, the larynx was displaced, and the ventral surface of the occipital bone was exposed. Two windows $(2 \times$ $3 \mathrm{~mm}$ ) were drilled through the occipital bone to expose the brainstem ventral surface. The meninges were left intact and there was no effusion of CSF. Once the ventral brainstem surface was exposed, a $473 \mathrm{~nm}$ laser (OptoDuet Laser; IkeCool) connected to a 200-nm-diameter optical fiber (IkeCool) was placed on the meninges. Laser power was set at $25 \mathrm{~mW}$. Timed photostimulations were delivered under the command of a pulse generator (Pulsemaster A300 Generator; WPI) connected to the laser power supply.

To find the position with the strongest response to laser stimulation in rats infected with SYN-ChR2-EYFP, the ventral surface was probed on one or both sides with $10 \mathrm{~s}$ laser pulses at $1 \mathrm{~min}$ intervals (four times in each location); the fiber was moved in a rectangular grid at 200 or $400 \mu \mathrm{m}$ step intervals (see Fig. 5). Once the most responsive site was identified, $100-150$ brief pulses stimulations were applied $(125 \mathrm{~ms}, 250 \mathrm{~ms}, 500 \mathrm{~ms}$, and $1 \mathrm{~s}$ duration) at $8 \mathrm{~s}$ intervals to test for a reset response.

Post hoc identification of neurons expressing EYFP allowed us to identify neurons that would have been activated during each experiment assuming a sufficient laser-induced photon flux. As shown in the results, viral injections were highly localized; the virus was not axonally transported and did not diffuse to other brainstem regions. Compared with local electrical stimulation, this experimental approach has the advantage of selectively activating neurons in a specific location (verified by postmortem EYFP expression analysis), excluding the possibility that axons of passage were responsible for the physiologically observed effects. Furthermore, laser stimulation gave us the possibility to excite neurons with both temporal and spatial precision, allowing us to study respiratory phase reset throughout the respiratory cycle. This is a major advantage compared with the results obtained with local application of drugs, either acting on inhibitory neurotransmission (i.e., the first part of this study), or on excitatory neurotransmission, or even via neuromodulation.

We used a lentivirus containing channelrhodopsin driven by the panneuronal marker synapsin to investigate and stimulate cells of the RTN/ pFRG independently from their neuronal phenotype. This approach was chosen since previous reports have shown that RTN Phox2b-expressing chemosensitive neurons in adult rats do not show late expiratory activity even in high respiratory drive stimulation (Abbott et al., 2009a; Kanbar et al., 2010; Marina et al., 2010); we therefore hypothesized that the broader RTN/pFRG contains not only these former subtypes that provide tonic excitatory drive to both the inspiratory and expiratory oscillators (Kanbar et al., 2010; Marina et al., 2010) but also key neurons (with an as-yetunknown phenotype) that are responsible for AE generation. These neurons may not be chemosensitive, may not express Phox $2 b$, or may express it in only low levels during adulthood.

Unit recordings. To record neuronal activity from the ventral surface of the brainstem, eight additional rats were anesthetized, implanted as described above, and positioned supine on the stereotaxic frame. The ventral surface of the brainstem was exposed as described above, and the meninges were ruptured. To record neuronal activity, either a tungsten electrode ( $5 \mathrm{M} \Omega$; A-M Systems) or a glass electrode ( $5 \mathrm{M} \Omega$; filled with $2 \%$ Neurobiotin in $0.5 \mathrm{~m} \mathrm{Na}$ acetate) was connected to a headstage (HZP; Grass Instruments) and the signal amplified and sampled at $10 \mathrm{kHz}$. Units were recorded adjacent to the site of injection of BIC/STRY (200 $\mathrm{nl} /$ each side). Injections were made $2.4 \mathrm{~mm}$ lateral from the midline and 
$0.8-1.2 \mathrm{~mm}$ caudal to the anterior inferior cerebellar artery. Units were recorded in similar position $\sim 2.3-2.5 \mathrm{~mm}$ from the midline.

Neurons recorded with Neurobiotin-filled glass electrodes were then stimulated with pulses of positive current $(10-20 \mathrm{~V}, 200 \mathrm{~ms}, 50 \%$ duty cycle for $10 \mathrm{~min}$ ) delivered through the recording electrode. At the end of the experiment, rats were perfused, brains collected, and the recording sites processed for immunohistochemistry (see below) to further identify morphology and location of the neurons.

A similar procedure was performed to record neuronal activity on laser stimulations after viral injections $(n=6)$. In this set of experiments, a tungsten electrode was positioned adjacent to the fiber. The tungsten electrode was positioned on the region where a maximal response on $10 \mathrm{~s}$ stimulation was achieved (see laser stimulation experimental protocol above). Single or trains of photostimulation were applied to identify neurons and test their response to laser stimulation.

Histology. At the end of each experiment, rats were transcardially perfused with $4 \%$ paraformaldehyde in phosphate buffer. The brains were collected, postfixed, and cryoprotected, and $40 \mu \mathrm{m}$ brainstem transverse sections were cut. Serial sections were either directly mounted on slides and coverslipped or immunoreacted for detection of specific neuronal markers. Immunohistochemistry was performed according to the following protocol. Free-floating sections were rinsed in PBS and incubated with $10 \%$ normal donkey antiserum (NDS) and $0.2 \%$ Triton X-100 in PBS for $60 \mathrm{~min}$ to reduce nonspecific staining and increase antibody penetration. Sections were incubated overnight with primary antibodies diluted in PBS containing 1\% NDS and $0.2 \%$ Triton X-100. The following day, sections were washed in PBS, incubated with the specific secondary antibodies conjugated to the fluorescent probes (Cy2-conjugated donkey anti-chicken; Cy3-conjugated anti rabbit; Cy3-conjugated antimouse; Jackson ImmunoResearch) diluted in PBS and 1\% NDS for $2 \mathrm{~h}$. Sections were further washed in PBS, mounted, and coverslipped with Fluorsave mounting medium (Millipore).

The primary antibodies used for this study were as follows: antibody to GFP (green fluorescent protein) (chicken; 1:1000; Aves Laboratories), Phox2b (rabbit; 1:500; kindly provided by Prof. J. F. Brunet, Centre National de la Recherche Scientifique, Paris, France), and to the neuronal marker NeuN (mouse; 1:500; Millipore).

For detection of Neurobiotin-filled neurons, sections were treated with $1 \% \mathrm{H}_{2} \mathrm{O}_{2}$ in PBS (10 min), washed in PBS, pretreated with Triton $\mathrm{X}-100(0.4 \%)$ for $30 \mathrm{~min}$, washed, and incubated in ABC (Vector Laboratories) for $2 \mathrm{~h}$. Labeling was revealed with nickel-enhanced diaminobenzidine solution (Vector Laboratories).

Slides were observed under an AxioCam2 Zeiss fluorescent microscope connected with AxioVision acquisition software or under a LSM510 Zeiss confocal microscope. Images were acquired, exported in TIFF files, and arranged to prepare final figures and identify electrode placement coordinates.

Data analysis and statistics. Traces were recorded on a computer and analyzed using Chart 7 Pro (ADInstruments), Excel, and Origin7 (OriginLab) software. The absolute value of EMG signals was digitally integrated with a time constant of $0.1 \mathrm{~s}$ to calculate peak amplitude and time onset. The flow signal was high-pass filtered to eliminate DC shifts and slow drifts, and used to calculate respiratory rate, period, inspiratory $\left(\mathrm{T}_{\mathrm{I}}\right)$ and expiratory $\left(\mathrm{T}_{\mathrm{E}}\right)$ durations. After filtering, the flow signal was digitally integrated to obtain tidal volume $\left(\mathrm{V}_{\mathrm{T}}\right)$ and minute ventilation $\left(\dot{\mathrm{V}}_{\mathrm{E}}\right)$.

For the purposes of analysis, AE was considered present when $\int A B D_{\text {EMG }}$ amplitude during the final $20 \%$ of the expiratory period was $>50 \%$ larger than in the first half (see Fig. $1 A$ ).

In BIC/STRY injection experiments, period, $\mathrm{T}_{\mathrm{I}}, \mathrm{T}_{\mathrm{E}}, \mathrm{V}_{\mathrm{T}}, \dot{\mathrm{V}}_{\mathrm{E}}$ and integrated peak amplitude of DIA $\mathrm{EMG}_{\mathrm{EMG}}, \mathrm{GG}_{\mathrm{EMG}}$, and $\mathrm{ABD}_{\mathrm{EMG}}$ were calculated for 100 cycles before injection and 100 cycles after either bilateral saline injection in control rats $(n=4)$ or the onset of AE in bilateral BIC/STRYinjected rats $(n=8)$.

In laser stimulation experiments, data were compared between respiratory cycles before (10) and during photostimulation. Changes in period, $T_{I}, T_{E}, V_{T}, \dot{V}_{E}$, and $\int E M G$ peak amplitude were calculated during the third set of photostimulation in the location where maximal responses occurred. Paired $t$ test (two-tail distribution) was used to determine statistical significance of changes before and after BIC/STRY injection or photostimulation. Significance was set at $p<0.05$. Data are shown as averaged values \pm SE.

Reset analysis. We analyzed the photostimulation-induced reset phenomenon across the respiratory cycle in seven SYN-ChR2-EYFPinjected rats. The beginning of each inspiratory cycle was marked by triggering on a threshold value of the respiratory flow. The average control respiratory period was calculated before stimulation trials and defined as spanning $0-360^{\circ}$ (i.e., from one inspiratory trigger to the next), with the onset of inspiration defined as phase $0^{\circ}$. Across respiratory cycles in our rats, inspiration (defined as the period of inward airflow) lasted from the trigger point at $0^{\circ}$ until $73.2-97.5^{\circ}$ ( $\min -\max$ values measured in $n=7$ experiments) while expiration was considered to take up the rest of the cycle. Thus, the phase of any event could be calculated based on the time at which it occurred relative to the ongoing respiratory period (specifically, the preceding inspiratory trigger) referenced to the average respiratory period. More specifically, phase values were obtained by computing the ratio of any onset or delay time to the respiratory period and multiplying by $360^{\circ}$.

In this way, we computed stimulus phase, induced phase, and the expected phase of respiration. Stimulus phase (i.e., the time at which stimulation was delivered during the ongoing respiratory cycle) was calculated based on the delay from the beginning of the previous inspiration to the onset of stimulus delivery. Induced phase (which was used to assess whether stimulation produced a systematic locking of the following cycle) corresponded to the delay from the laser onset to the subsequent inspiration. This latter value was compared with the expected phase (which was simply calculated assuming that stimulation had no effect) and was computed as the difference between the presumed end of the respiratory period as calculated from the previous inspiration (i.e., $360^{\circ}$ ) and the phase at which stimulation occurred (i.e., $360^{\circ}$-stimulus phase). Examples of how these values were taken are shown graphically in Figure $6 D$.

Phase measurements for each experimental condition were binned across the full $360^{\circ}$ cycle in increments of $18^{\circ}$ (totaling 20 bins) and were analyzed using circular statistics to test for uniformity using the Raleigh $z$ distribution and to compute an average phase angle. The average phase angle for any given dataset was characterized by a vector with an angle equal to the average phase and with a length (normalized, $0-1$ ) related to the angular dispersion of the individual phase values (length $=1$ being no dispersion). Circular statistics were also applied across each average vector to compute a grand average vector across all experiments (again having both an angle and normalized length) and also to test for nonuniformity, using the Raleigh $z$ distribution. Phase measurements were obtained using custom software written in Matlab, version 7.4 (The MathWorks). Results were plotted using Origin 7 software.

\section{Results}

Active expiration is absent in normocapnic or hypercapnic, anesthetized, vagotomized rats

Forced expiratory flow (i.e., AE) corresponds to an increase in outward airflow in the late expiratory phase immediately before inspiration, because of recruitment of expiratory muscle groups including $\mathrm{ABD}, \mathrm{GG}$, and internal intercostals. Overall, $\mathrm{AE}$ increases tidal (inspired) volume $\left(\mathrm{V}_{\mathrm{T}}\right)$ since lung inflation begins at a smaller end-expiratory lung volume. We considered AE as being present when $\int \mathrm{ABD}_{\mathrm{EMG}}$ amplitude during the final $20 \%$ of the expiratory period was $>50 \%$ larger than in the first half. Examples of respiratory cycles without and with $\mathrm{AE}$ are shown in Figure $1 A$.

During baseline conditions in our urethane-anesthetized and vagotomized adult rats, expiration was consistently passive. During passive expiration, outward (expiratory) airflow quickly peaked and then rapidly declined, a consequence of the passive deflation of the lung and chest wall to resting from stretched positions at the end of inspiration. Passive expiration was characterized by tonic abdominal muscle electromyogram $\left(\mathrm{ABD}_{\mathrm{EMG}}\right)$ activity either with or without (phasic) inhibition during inspiration (Fig. 1A). We found no instances of $\mathrm{AE}$ in these rats 
A
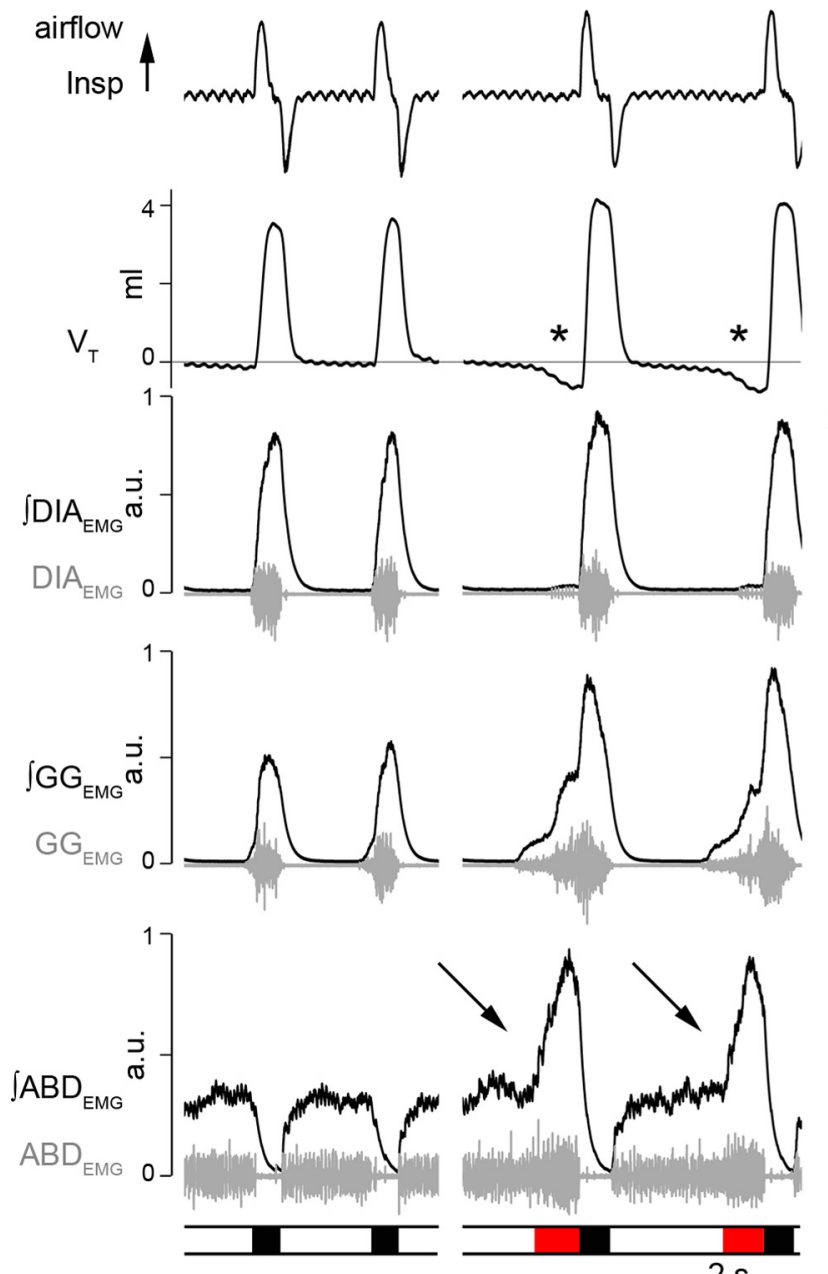
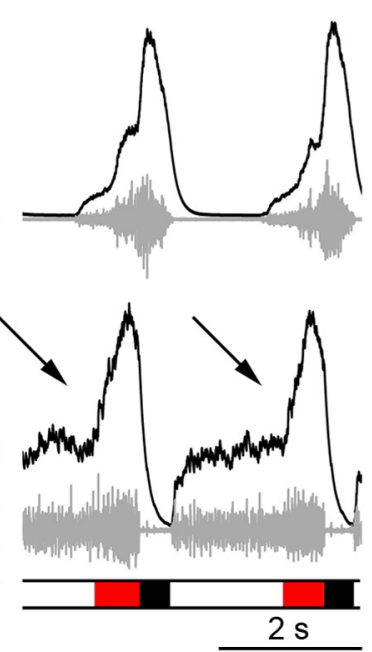

B

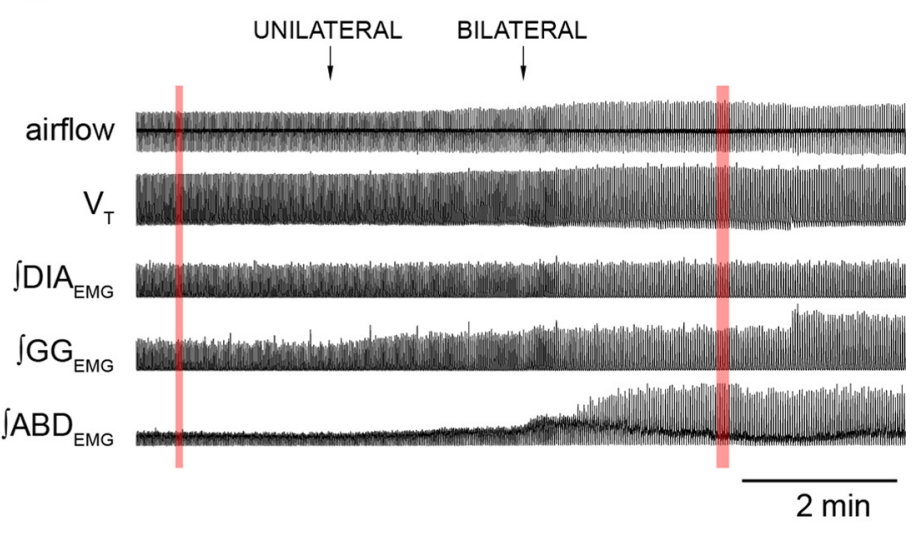

C

airflow AHH $\sim$ HWHAt

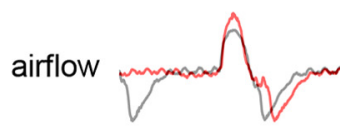

$\mathrm{V}_{\mathrm{T}}$

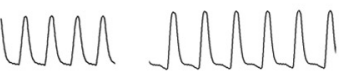

JDIA

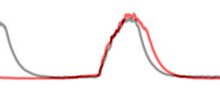

$\int D I A_{\text {eMG }}$

una hunn

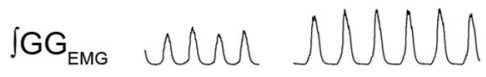

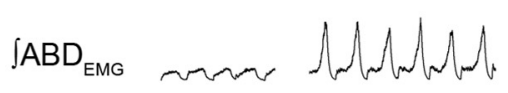

$5 \mathrm{~s}$

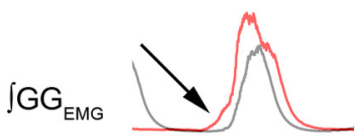

$\int_{A B D} D_{\text {EMG }}$

$2 s$

Figure 1. Local application of BIC/STRY in RTN/pFRG induces long-lasting AE. $A$, Respiratory airflow, $\mathrm{V}_{\mathrm{T}}, \mathrm{DIA}_{\mathrm{EMG}}, \mathrm{GG}_{\mathrm{EMG}}, \mathrm{ABD}_{\mathrm{EMG}}$ (gray), and their integrals (black) during passive expiration (left) in respiratory cycles at rest and during $A E$ on stimulation (right). The bar at bottom indicates inspiration (black) and $A E$ (red). During $A E$, $\int A B D_{E M G}$ and $\int G G_{E M G}$ increased and $V_{T}$ dipped below resting levels (asterisks). Integrated traces are plotted from minimum to maximum and scaled $[0,1]$. Note the potent increase of $\int A_{B D} D_{E M G}$ at the end of expiratory period during $A E$. $B$, Response to unilateral and bilateral application of BIC/STRY (50 $\mu \mathrm{m} ; 200 \mathrm{nl}$ ) in RTN/pFRG. The traces in red boxes are expanded in C. D, Superimposed traces of airflow and EMGs before (black) and after (red) BIC/STRY application. The arrow in $D$ indicates effect of BIC/STRY on $\mathrm{GG}_{\mathrm{EMG}}$ onset.

breathing room air and at normal end-expiratory $\mathrm{CO}_{2}$ levels $(\sim 5 \% ; n=20)$. $\mathrm{ABD}_{\mathrm{EMG}}$ was consistently tonic between inspirations and inhibited during inspiration. Furthermore, $\mathrm{GG}_{\mathrm{EMG}}$ was inspiratory-modulated, beginning $\sim 80 \mathrm{~ms}$ before diaphragmatic EMG (DIA $\mathrm{EMG}_{\text {G }}$ ) onset. Respiratory period was $1.7 \pm 0.1 \mathrm{~s}$ and inspiratory duration $\left(\mathrm{T}_{\mathrm{I}}\right)$ was $0.4 \pm 0.1 \mathrm{~s}(n=8)$.

Adding $5 \% \mathrm{CO}_{2}$ to the inspired gas mixture increased ventilation at $5 \mathrm{~min}$ by $\sim 15 \%$ but did not trigger AE. Peak $\int \mathrm{DIA}_{\mathrm{EMG}}$ and $\int \mathrm{GG}_{\mathrm{EMG}}$ amplitudes increased by $15.0 \pm 3.9 \%(p=0.02)$ and by $29.8 \pm 4.1 \%(p=0.001)$. $\mathrm{V}_{\mathrm{T}}$ increased by $9.1 \pm 2.6 \%$ $(p=0.006)$ and minute ventilation $\left(\dot{\mathrm{V}}_{\mathrm{E}}\right)$ by $15.8 \pm 2.7 \%(p=$ $0.003)$; respiratory frequency increased because of shortening of both inspiration $(-11.0 \pm 1.6 \% ; p=0.001)$ and expiration $(-2.5 \pm 1.9 \% ; p=0.006)$.

\section{Bicuculline/strychnine microinjections in RTN/pFRG trigger AE}

We hypothesized that the RTN/pFRG does not generate AE at rest because of suppression by synaptic inhibition. Consistent with this idea, bilateral injection in the RTN/pFRG of antagonists for GABAergic and glycinergic transmission (BIC/STRY, $50 \mu \mathrm{M}$ ) triggered $\mathrm{AE}$, as demarked by bursts of $\mathrm{ABD}_{\mathrm{EMG}}$ and $\mathrm{GG}_{\mathrm{EMG}}$ during late expiration (Fig. $1 B-D$ ). Just before the late expiratory burst, $\mathrm{ABD}_{\mathrm{EMG}}$ was tonic with duration similar to the total duration of expiration before BIC/STRY (average difference, $10.0 \pm$ $6.7 \% ; p=0.2 ; n=8)$. During $\mathrm{AE}, \mathrm{ABD}_{\mathrm{EMG}}$ burst duration was $0.40 \pm 0.05 \mathrm{~s}$, with significantly longer expiration (43.8 $\pm 7.1 \%$; $\left.p=5 \times 10^{-5}\right)$ compared with controls. With a smaller absolute decrease in $\mathrm{T}_{\mathrm{I}}\left(-20.2 \pm 3.3 \% ; p=2 \times 10^{-3}\right)$, the respiratory period increased from $1.6 \pm 0.1$ to $1.9 \pm 0.1 \mathrm{~s}\left(p=2 \times 10^{-4}\right)$. $\mathrm{V}_{\mathrm{T}}$ increased by $23.2 \pm 3.2 \%\left(p=3 \times 10^{-4}\right)$ but $\dot{V}_{\mathrm{E}}$ did not change $\left(\Delta \dot{\mathrm{V}}_{\mathrm{E}},-3.4 \pm 4.5 \% ; p=0.53\right)$ (Fig. $\left.2 \mathrm{~A}\right)$. These effects were in contrast to those for saline injections that produced neither AE nor any significant changes in period $(p=0.58), \mathrm{V}_{\mathrm{T}}(p=0.23)$, or DIA $(p=0.33), \mathrm{GG}(p=0.59)$, and $\mathrm{ABD}(p=0.57) \mathrm{EMG}$ $(p>0.05 ; n=4)$.

During cycles with $\mathrm{AE}, \mathrm{ABD}_{\mathrm{EMG}}$ continued to be inhibited during inspiration, and $\mathrm{GG}_{\mathrm{EMG}}$ developed two distinct phases: inspiratory (concomitant with $\mathrm{DIA}_{\mathrm{EMG}}$ ) and expiratory (concomitant with $\mathrm{ABD}_{\mathrm{EMG}}$ ). Peak $\int \mathrm{ABD}_{\mathrm{EMG}}$ amplitude increased by $3056 \pm 977 \%$ (measured relative to tonic activity levels in the preinjection period; $p=0.04$ ). The peak amplitude of $\int \mathrm{DIA}_{\mathrm{EMG}}$ 
A
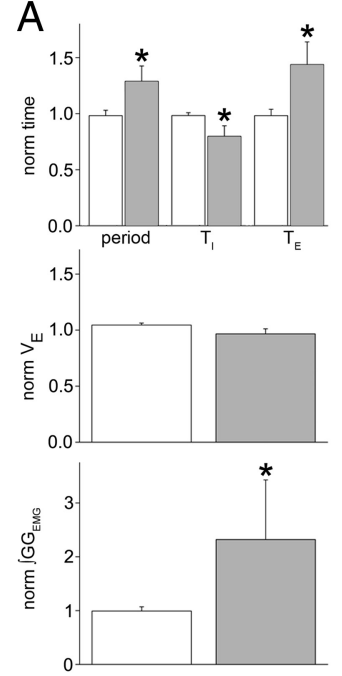
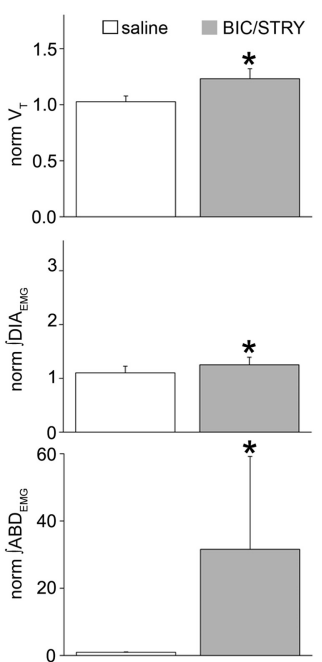

B

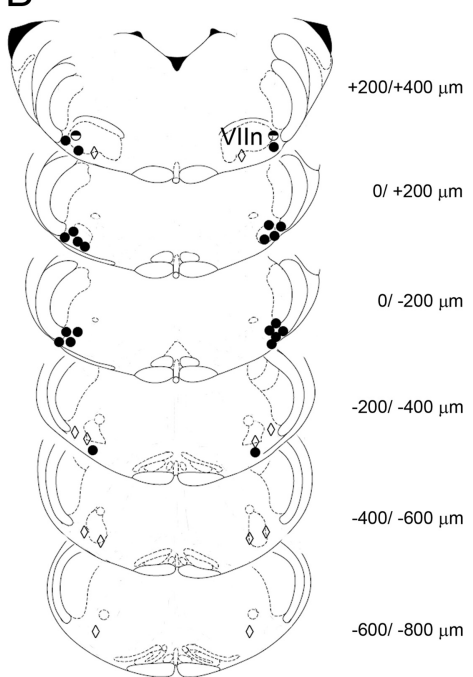

Figure 2. RTN/pFRG BIC/STRY injections induced AE. $A$, Respiratory period, $\mathrm{T}_{\mathrm{l}}, \mathrm{T}_{\mathrm{E}}, \mathrm{V}_{\mathrm{T}}, \dot{\mathrm{V}}_{\mathrm{E},} \int \mathrm{SIA}_{\mathrm{EMG}} \int \mathrm{GG}_{\mathrm{EMG}} \int \mathrm{ABD}_{\mathrm{EMG}}$ calculated from 100 cycles before BIC/STRY injection and after AE onset. Values averaged and normalized in each experiment $(n=4$ saline, white; $n=8 \mathrm{BIC} / \mathrm{STRY} ; \mathrm{gray})$. The asterisk $\left({ }^{*}\right)$ indicates statistical significance $(p<0.05)$ between preinjection and postinjection. Error bars indicate SEM. B, Representative sections of adult rat brainstem [modified from Paxinos and Watson (1998)]; $0 \mu \mathrm{m}$ is caudal tip of VIIn. Each symbol represents an injection site. The circles identify locations where AE was induced strongly (filled) or weakly (half-filled). The diamonds identify locations that did not induce AE.

and of the inspiratory component of $\int \mathrm{GG}_{\mathrm{EMG}}$ increased by $25.2 \pm 6.2 \%(p=0.01)$ and $132.1 \pm 39.4 \%(p=0.005)$. The onset of the $\int G_{\mathrm{EMG}}$ preceded DIA $\mathrm{EMG}_{\mathrm{EM}}$ onset by $194 \pm 17 \mathrm{~ms}$ during $\mathrm{AE}$ compared with $82 \pm 16 \mathrm{~ms}$ prior in control periods (Fig. 1).

AE was observed during every cycle for $9.0 \pm 1.2 \min (n=8)$ after bilateral BIC/STRY injection and thereafter was intermingled with respiratory cycles without $\mathrm{AE}$. After the cessation of AE, tonic levels of $\mathrm{ABD}_{\mathrm{EMG}}$ and $\mathrm{GG}_{\mathrm{EMG}}$ were augmented compared with the control period for an additional 5-7 min.

The most effective injection sites, based on histological examination, were at the lateral edge of VIIn, $\pm 200 \mu \mathrm{m}$ from its caudal tip (Fig. $2 B$ ). Injections $>400 \mu \mathrm{m}$ from the caudal tip of VIIn failed to induce $\operatorname{AE}(n=5)$. Interestingly, the responses from more medial positions $(n=6)$ were either weaker or only developed after a long delay. This suggests that the site for AE induction by disinhibition was lateral to the VIIn, which is distinctly lateral to chemosensitive adult rat RTN neurons (Mulkey et al., 2004; Stornetta et al., 2006; Guyenet et al., 2008).

To determine the effects of BIC/STRY on RTN/pFRG neurons, we used a ventral approach (see Materials and Methods) to record while injecting BIC/STRY, and a higher concentration of BIC/STRY $(250 \mu \mathrm{M})$ for a stronger, more prolonged effect (16.2 \pm 1.3 min duration; $n=8 ; 15$ trials). Before ejection, we were unable to record late expiratory-modulated neurons. Rather, we observed neurons with inspiratory $(n=18)$, tonic expiratory $(n=4)$, or nonrespiratory (i.e., tonic) $(n=11)$ patterns of activity. In contrast, on activation of AE by BIC/STRY application, some neurons fired with late expiratory activity in phase with $\mathrm{ABD}_{\mathrm{EMG}}$ bursts $(n=9)$ (Fig. $3 A$ ). Interestingly, at the end of the BIC/STRY-induced AE activation, in instances in which $\mathrm{AE}$ cycles were interspersed with no $\mathrm{AE}$ (i.e., inspirationonly) cycles, late expiratory neuronal activity was present only during the former $(n=3)$ (Fig. $3 B)$.

$\mathrm{LL}_{\mathrm{EMG}}$ had a late expiratory and an inspiratory component, similar to $\mathrm{GG}_{\mathrm{EMG}}$ (Fig. 1A). Therefore, based on their different firing patterns, we differentiated between late expiratory RTN/pFRG $(n=9)$ neurons and adjacent VIIn $(n=6)$ motoneurons that were active during both late expiration and inspiration. Juxtacellular labeling of recorded neurons with Neurobiotin confirmed this classification. Neurons with only late expiratory activity were outside the VIIn, close to the medullary ventral surface $(235 \pm 46 \mu \mathrm{m}$ from the surface; $n=4$ ) and $2.36 \pm 0.02 \mathrm{~mm}$ from the midline, with soma diameters of $26.2 \pm 1.0 \mu \mathrm{m}$ (Fig. 3D). VII motoneurons were larger $(33.4 \pm 2.6 \mu \mathrm{m} ; n=5)$ and were embedded within the VIIn (2.0 $\pm 0.1 \mathrm{~mm}$ from the midline and $820 \pm 156 \mu \mathrm{m}$ from the ventral surface) (Fig. 3E).

\section{Viral injection: histology}

In the interest of exploring our hypothesis that the RTN/pFRG is critical for the generation of $\mathrm{AE}$, we focused our viral injections to transfect RTN/pFRG neurons with ChR2 in the region where BIC/STRY induced the strongest AE (i.e., ventrolateral to the caudal pole of VIIn). We used a lentivirus coding for the mutated ChR2 protein (ChR2-H134R) fused with EYFP driven by the neuronal specific promoter SYN [SYN-ChR2-EYFP (Adamantidis et al., 2007; Gradinaru et al., 2007)].

Based on histological examination of sections from injected rats, neurons expressing $\operatorname{EYFP}\left(\mathrm{EYFP}^{+}\right)$were ventrolateral to the caudal pole of VIIn, in the region homologous to perinatal rat pFRG (Onimaru and Homma, 2003) and embryonic mouse parafacial oscillator (e-pF) (Thoby-Brisson et al., 2009). EYFP ${ }^{+}$ neurons were $2.2-2.5 \mathrm{~mm}$ from the midline and close to the ventral brainstem surface (Fig. $4 A-C$ ). More medially were only a few, if any, $\mathrm{EYFP}^{+}$neurons.

In the viral injection site core $( \pm 320 \mu \mathrm{m}$ rostral/caudal to the caudal pole of VIIn), $47.8 \pm 9.1$ neurons/slice ( $40 \mu \mathrm{m}$ thick) were observed, whereas outside this core the $\mathrm{EYFP}^{+}$neuron density rapidly decreased (Fig. $4 G-I)(n=4)$. Less than $10 \%$ of the EYFP $^{+}$neurons/slice $(9.4 \pm 1.8 ; n=4)$ had the morphology and location of facial motoneurons, and they were mainly $0-500 \mu \mathrm{m}$ rostral to the caudal pole of VIIn. Relatively few $\mathrm{EYFP}^{+}$neurons $(8.7 \pm 3.7$ neurons/slice in the core of the injection; $n=3)$ expressed Phox $2 \mathrm{~b}$, the marker for chemically coded RTN neurons (Stornetta et al., 2006); these neurons were mainly located ventral and medial to the caudal pole of VIIn.

With the exception of the injection site and its immediate surround, we did not observe EYFP ${ }^{+}$neurons in the brainstem. EYFP $^{+}$-filled fibers extended along the ventral respiratory column in the Bötzinger complex (BötC), preBötC, and, more caudally, in the rostral and caudal ventral respiratory groups. In addition, $\mathrm{EYFP}^{+}$-filled fibers projected toward and surrounded the hypoglossal and the solitary tract (NTS) nuclei. The presumptive origin of these fibers was $\mathrm{EYFP}^{+}$neurons at the injection site (Fig. $4 D-F$ ).

\section{Laser stimulation of SYN-ChR2-EYFP-expressing RTN/pFRG neurons induced AE}

Four weeks after viral injection, we reanesthetized the rats to use a ventral approach to photostimulate $(473 \mathrm{~nm})$. We initially 
A
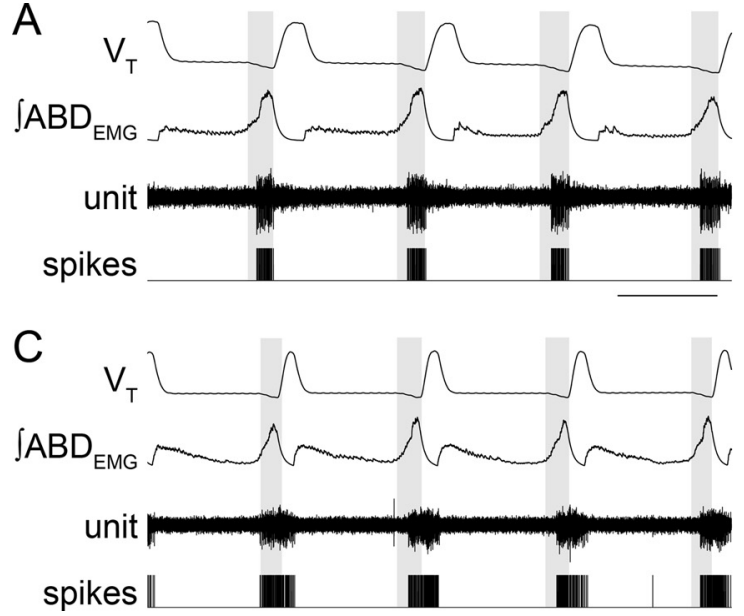

B

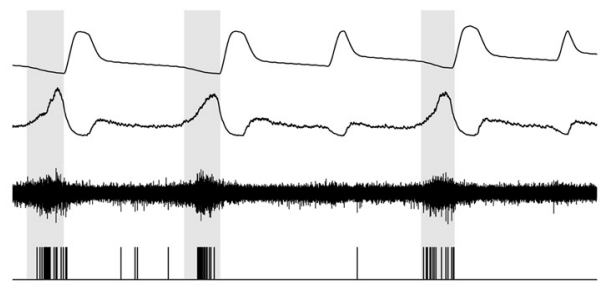

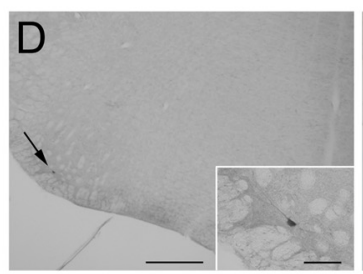

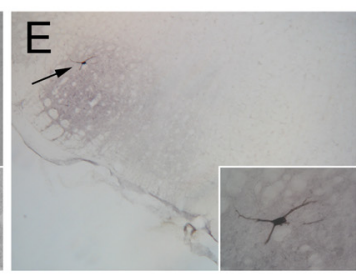

Figure 3. RTN/pFRG and VII motor neuronal activity during AE. $A-C$, Three different neurons that were silent at rest, and rhythmic after induction of $A E$. Traces (top to bottom), $V_{T}, \int A B D_{E M G}$ neuronal activity, and derived spikes. The shaded boxes demark late expiration. Calibration: $2 \mathrm{~S}$. D, E, Photomicrographs of transverse sections of rat brainstem illustrating juxtacellular labeling of late expiratory neuron recorded shown in $\boldsymbol{B}(\boldsymbol{D}$, arrow) and VII motoneuron ( $\boldsymbol{E}$, arrow). The insets show higher magnification of neurons. Scale bars: $\boldsymbol{D}, \boldsymbol{E}, 500 \mu \mathrm{m} ; \boldsymbol{D}, \boldsymbol{E}$, insets, $100 \mu \mathrm{m}$.

tested the response to continuous photostimulation (10 s), mapping the response by moving the optical fiber in $400 \mu \mathrm{m}$ rostrocaudal steps. Photostimulation was most effective at injection sites (demarked by post hocidentification) close to the caudal pole of VIIn, $2.3-2.5 \mathrm{~mm}$ from the midline, with progressively weaker responses as we moved away (Fig. 5). Photostimulation at the injection site changed $\mathrm{ABD}_{\mathrm{EMG}}$ from tonic $(n=$ 8) [i.e., constant activity during the expiratory phase (with no change or decreased activity during the inspiratory phase) (Sears et al., 1982)] to incrementing late expiratory bursts of activity (i.e., AE). In several experiments, within the $10 \mathrm{~s}$ continuous photostimulation period, the first respiratory cycles had larger $\mathrm{ABD}_{\mathrm{EMG}}$ compared with the last ones $(n=6)$. Concomitant with the $\mathrm{ABD}_{\mathrm{EMG}}$ burst, the late expiratory $\mathrm{GG}_{\mathrm{EMG}}$ component clearly increased (Fig. 5).

Unilateral photostimulation $(n=6)$ in SYN-ChR2-EYFP-injected rats generated a moderate increase in peak inspiratory flow $(8.3 \pm 3.0 \% ; p=0.02)$ and $\mathrm{V}_{\mathrm{T}}(2.1 \pm 0.9 \%$; $p=0.03)$ compared with prestimulation values. Respiratory period $(-5.9 \pm 4.3 \%$; $p=0.11)$ and $\dot{V}_{\mathrm{E}}(9.9 \pm 4.3 \% ; p=0.06) \mathrm{did}$ not change significantly. Contralateral peak $\int \mathrm{ABD}_{\mathrm{EMG}}$ increased by $72.7 \pm 10.9 \%(p=$ $\left.5 \times 10^{-4}\right)$ and ipsilateral peak $\int G_{\mathrm{EMG}}$ increased by $62.3 \pm 12.1 \%(p=0.007)$; peak $\int \mathrm{DIA}_{\mathrm{EMG}}$ increased slightly $(+5.4 \pm 2.2 \%$; $p=0.03$ ).

Bilateral photostimulation $(n=5)$ produced similar but larger effects: peak respiratory flow, $\mathrm{V}_{\mathrm{T}}$, and $\dot{\mathrm{V}}_{\mathrm{E}}$ increased by $17.0 \pm 4.2(p=0.008), 5.6 \pm 2.0 \%(p=0.03)$, and $20.3 \pm$ $7.4 \%(p=0.03)$, respectively; respiratory period decreased by $12.6 \pm 5.9 \%(p=0.02)$. Peak $\int \mathrm{ABD}_{\mathrm{EMG}}$ and $\int \mathrm{GG}_{\mathrm{EMG}}$ increased by $188.1 \pm 41.7 \%(p=0.005)$ and $100.7 \pm 19.1 \%$ $(p=0.02)$, respectively. Peak $\int \mathrm{DIA}_{\mathrm{EMG}}$ increased slightly $(5.0 \pm 2.5 \% ; p=0.07)$.
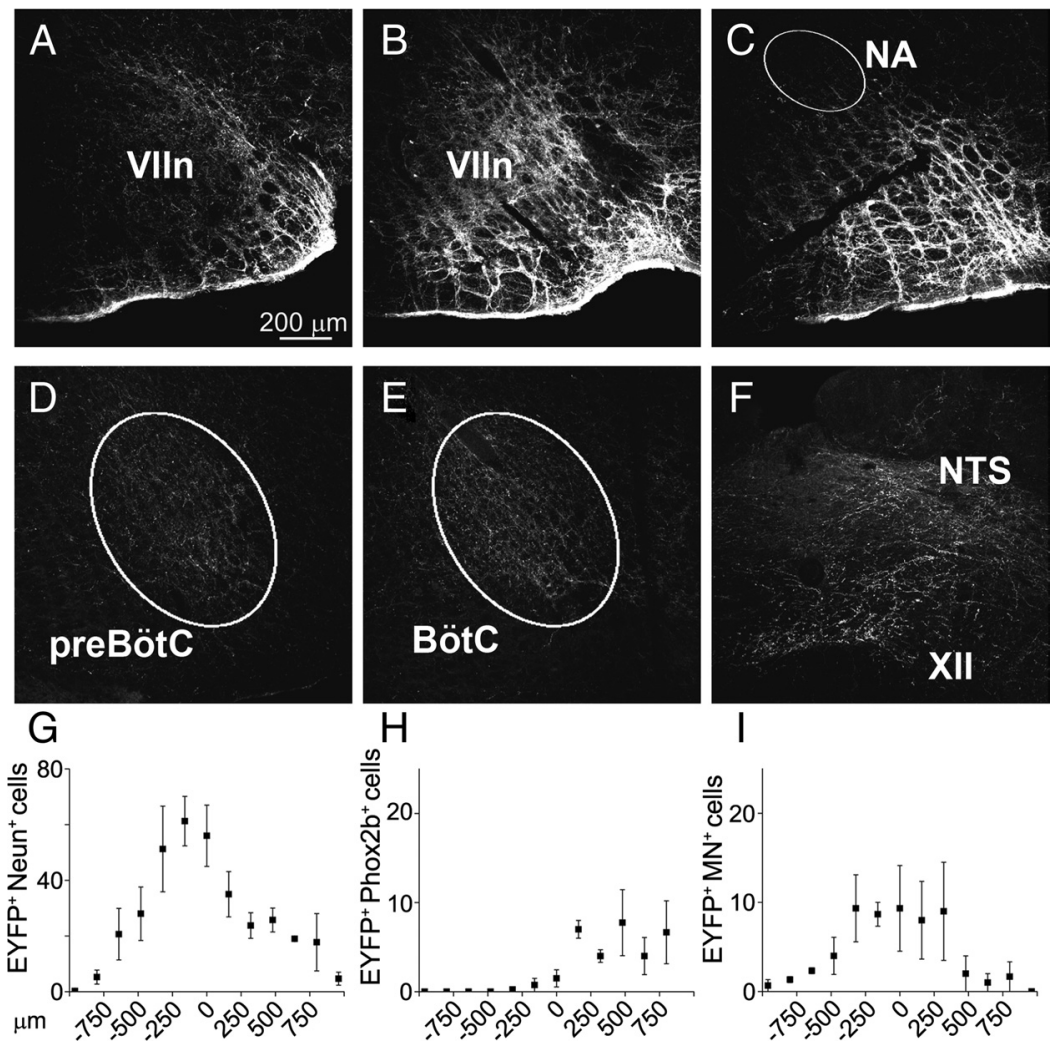

Figure 4. EYFP expression 4 weeks after SYN-ChR2-EYFP lentivirus injected in rat RTN/pFRG. $\boldsymbol{A}$-C, EYFP expression in cell bodies and fibers surrounding rostral VIIn, caudal VIIn, and caudal to the VIIn, respectively. D-F, EYFP expression in fibers in preBötC, BötC, hypoglossal nucleus (XII), and NTS (NA, nucleus ambiguus). G-I, Rostrocaudal distribution of EYFP ${ }^{+} / \mathrm{NeuN}^{+}, \mathrm{EYFP}^{+} / \mathrm{Phox} \mathrm{b}^{+}$, and EYFP ${ }^{+} / \mathrm{MN}^{+}$double-labeled somata in relationship to caudal pole of the VIIn $(n=4)$.

Rats in which viral expression was low or in which SYNEYFP virus was expressed (as a control) did not respond to photostimulation.

\section{Resetting of respiratory rhythm}

During these trials, the respiratory cycle subsequent to photostimulation onset appeared to begin at a relatively consistent interval, suggesting resetting of the respiratory rhythm. To explore this observation, we used brief (125-1000 ms) photostimulation 
A

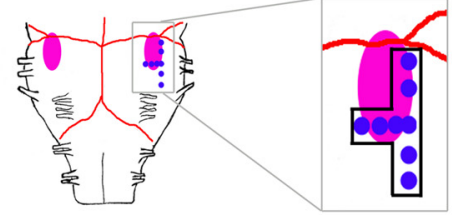

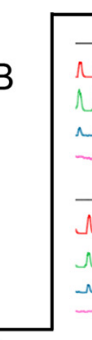

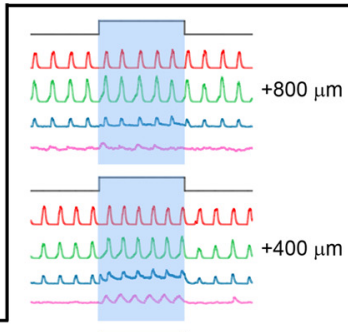

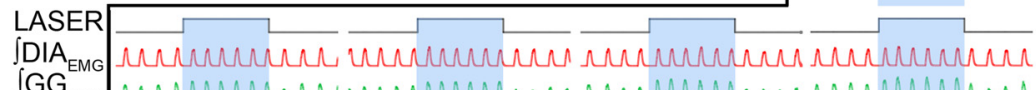

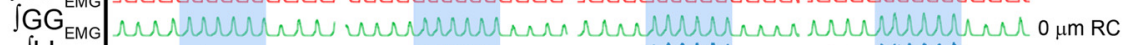

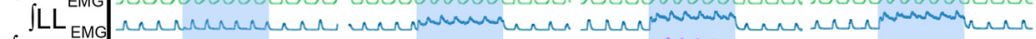

$\int A B D_{\text {EMG }}$
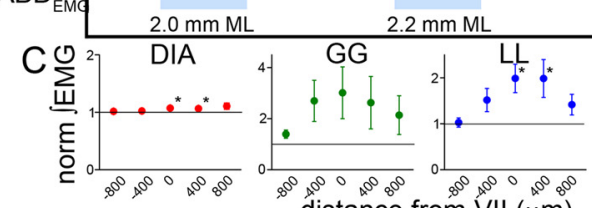

$2.4 \mathrm{~mm} \mathrm{ML}$
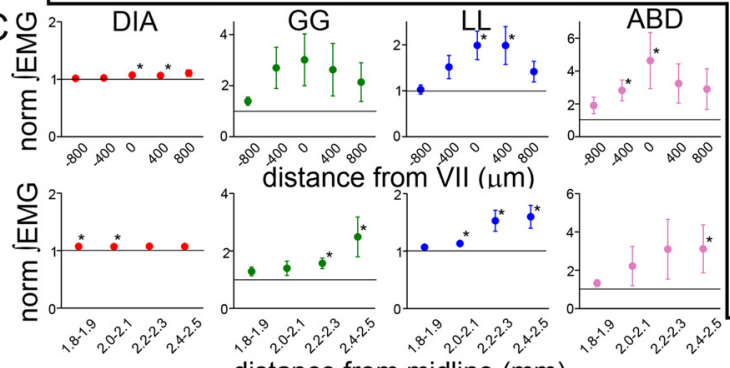

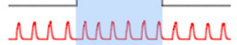

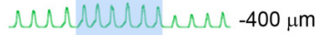

aranumentranar

romennom MULHULINAL Nuluburnh $-800 \mu \mathrm{m}$

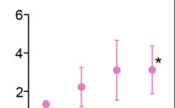

crarenararara  $2.5 \mathrm{~mm} \mathrm{ML}$ distance from midline (mm)

Figure 5. Photostimulation on ventral medullary surface over RTN/pFRG in SYN-ChR2-EYFP-injected rats induced AE. $\boldsymbol{A}$, Diagram of ventral brainstem surface (VIIn: pink) indicating photostimulation sites (blue dots correspond to sets of traces in $\boldsymbol{B}$ ). $\boldsymbol{B}$, Effects of 10 s photostimulus (top trace) on DIA $A_{E M G}$ (red), $\mathrm{GG}_{E M G}$ (green), $\mathrm{LL}_{E M G}$ (blue), and $\mathrm{ABD}_{\mathrm{EMG}}$ (pink). Right, $\pm 400 \mu \mathrm{m}$ rostrocaudal (RC) intervals; middle, $\pm 200 \mu \mathrm{m}$ mediolateral $(\mathrm{ML})$ intervals. $C$, Effect of 10 s photostimulus along the rostrocaudal (top; $n=5$ ) and mediolateral (bottom; $n=4$ ) axes on peak DIA $A_{E M G}\left(\right.$ red), $\mathrm{GG}_{\mathrm{EMG}}$ (green), $\mathrm{LL}_{\mathrm{EMG}}$ (blue), and $\mathrm{ABD}_{\mathrm{EMG}}$ (pink). The horizontal lines represent normalized control values before photostimulation. The asterisks indicate $p<0.05$ between control and photostimulation.

pulses applied at different phases of the respiratory cycle and found that, regardless of the stimulus phase, the induced poststimulus respiratory phase fell into a narrow range when aligned with respect to the onset of stimulation (Fig. 6A).

Together with respiratory reset, stimulation evoked $\mathrm{ABD}_{\mathrm{EMG}}$ and $\mathrm{GG}_{\mathrm{EMG}}$ bursts coincident with inhibition in $\mathrm{DIA}_{\mathrm{EMG}}$ just before subsequent $\mathrm{DIA}_{\mathrm{EMG}}$ onset and inspiratory flow (Fig. $6 B$ ). The average delay in $\mathrm{ABD}_{\mathrm{EMG}}$ and $\mathrm{DIA}_{\mathrm{EMG}}$ onset from onset of photostimulation was $115 \pm 13$ and $886 \pm 52 \mathrm{~ms}$, respectively $(n=7)$ (Fig. $6 B$ ). Concomitant with this stimulus-locked $\mathrm{ABD}_{\mathrm{EMG}}$ activity was a significant increase in expiratory flow (i.e., $\mathrm{AE}$ ) before the next inspiration (Fig. $6 C$, arrow). After $\mathrm{AE}, \mathrm{T}_{\mathrm{I}}$ was shorter $(-11.8 \pm 2.1 \%$; $p=$ 0.005 ) and peak inspiratory flow increased compared with preceding nonstimulated cycles $(16.4 \pm 1.5 \% ; p=0.004)$. If stimulation occurred during inspiration, the $\mathrm{DIA}_{\mathrm{EMG}}$ burst was interrupted, concurrent with the onset of an $\mathrm{ABD}_{\mathrm{EMG}}$ burst, indicating a disruption and subsequent reset of the respiratory cycle.

To systematically analyze the reset, we determined the phase values for both the onset of stimulation relative to the ongoing respiratory cycle (stimulus phase) and the onset of the subsequent inspiration (induced phase), shown diagrammatically in green in Figure $6 D$. By plotting these values and using circular statistics (Zar, 1996), we could determine whether either or both were randomly distributed across the cycle or were clustered at a preferred phase (Fig. 6E). Consistent with the random delivery of stimuli across all phases of the respiratory cycle, the stimulus phase distribution (red dots) appeared uniform (Raleigh $z: p=$ 0.52 ). However, the distribution of induced phase values (green dots) showed a marked clustering suggestive of stimulus-induced phase reset. Indeed, this distribution was significantly nonuniform (Raleigh $z: p<10^{-35}$ ), and the average phase was repre- sented by a vector (see Material and Methods) having a phase angle of $78^{\circ}$ and normalized length (radius) of 0.90 (Fig. $6 E$, green vector). Vector lengths close to 1 are indicative of low dispersion of individual angular values as well as significant phase preferences (i.e., nonuniformity) in polar distributions (Zar, 1996). For all experiments $(n=7)$, the distributions of induced phase were significantly different from uniformity (using Raleigh tests) and are shown as individual vectors across in Figure $6 F$. Notably, these averages clustered in the top left quadrant (range, 80 $150^{\circ}$ ), and their cumulative distribution was also nonuniform (Raleigh $z$ : $p=7 \times$ $10^{-3}$ ). The overall (grand) average across all experiments had a phase angle of $120^{\circ}$ with a normalized radius of 0.80 (Fig. $6 \mathrm{~F}$, black line). Thus, phase reset by RTN/ pFRG photostimulation was consistent and significant both within and across experiments. (For additional analysis discussion of phase reset, see supplemental information, Phase reset analysis; supplemental Fig. S1, available at www.jneurosci. org as supplemental material.)

\section{Unit recordings of \\ light-activated neurons}

The previous results could have been attributable to the activation of an (1) expiratory oscillator at the stimulation site, which we hypothesized (Janczewski and Feldman, 2006); or (2) oscillator at another site via projections from the RTN/pFRG. In the first hypothesis and given the observation that we did not observe any expiratorymodulated neurons before stimulation, an essential prediction is that some tonic/silent RTN/pFRG neurons become rhythmically active concurrent with or before the onset of AE. In the latter case, tonic neurons could simply become more active, as it is the case with RTN chemosensitive neurons (Abbott et al., 2009a). Thus, we recorded single-unit activity at the sites most sensitive to photostimulation.

In rats injected with SYN-ChR2-EYFP $(n=6)$ and in the absence of photostimulation, we failed to find RTN/pFRG neurons with late expiratory activity. Rather, neurons were either inspiratory-modulated or tonically active, the latter being characterized by irregular firing uncorrelated with the respiratory rhythm $(n=12)$. To search for neurons that responded to photostimulation, we applied brief ( $<30 \%$ of expiratory time) light pulses while probing for neuronal activity (Fig. $7 A$ ). Once an activated neuron was found, $10 \mathrm{~s}$ stimuli that induced $\mathrm{AE}$ were applied; the majority of responsive neurons were tonically activated or only weakly modulated $(n=15 / 19)$. Approximately $20 \%$ ( 4 of 19) of the neurons became expiratory-modulated ( $n=$ 4) (Fig. $7 B, C$ ) producing a late-expiratory burst of activity concurrent with the late expiratory burst of $\mathrm{ABD}_{\mathrm{EMG}}$. Trains of $10 \mathrm{~ms}$ pulses at $20 \mathrm{~Hz}$ for $10 \mathrm{~s}$ could also induce late expiratory activity in these neurons (Fig. 7C). Thus, regardless of whether the stimulus was continuous or brief, a subpopulation of RTN/pFRG neurons became rhythmically active during the late expiratory phase. 


\section{Discussion}

We provide evidence consistent with the hypothesis that in adult rats a conditional RTN/pFRG oscillator generates AE. This oscillator is characterized at rest by silent neurons that when properly stimulated become rhythmic.

In perinatal rodents in vitro, the predominant firing phenotype of pFRG neurons is preinspiratory, bursting before and after inspiration (Onimaru et al., 1995, 2006; Onimaru and Homma, 2003). In addition, a subset of perinatal rhythmically active pFRG neurons are chemosensitive and some express Phox2b (Onimaru et al., 2008). In contrast, in adult anesthetized rats, chemosensitive Phox $2 \mathrm{~b}^{+}$RTN neurons are not rhythmic (Mulkey et al., 2004; Stornetta et al., 2006). In rodents, the fetal embryonic parafacial group (Thoby-Brisson et al., 2009), the neonatal/juvenile pFRG (Janczewski et al., 2002; Onimaru and Homma, 2003; Onimaru et al., 2008), and the adult RTN (Guyenet et al., 2008) may be part of the same neuronal network in different developmental stages (Feldman et al., 2009; Thoby-Brisson et al., 2009), or alternatively, they may represent overlapping neuronal populations that contribute to respiratory rhythms in different ways through development.

We hypothesized that, in adult anesthetized vagotomized rats, the RTN/ pFRG conditional oscillator responsible for the generation of $\mathrm{AE}$ is functional but effectively silenced by endogenous inhibition. Indeed, AE could be induced by local microinjection of GABAergic and glycinergic antagonists as summarized in supplemental Figure S2 (available at www. jneurosci.org as supplemental material). This manipulation transformed silent RTN/pFRG neurons to late expiratory modulation that was concurrent with AE. The inhibition of RTN/pFRG rhythmicity could originate in respiratory-modulated GABAergic and glycinergic neurons in the ventral respiratory column, in particular from the BötC and preBötC (Cream et al., 2002; Rosin et al., 2006; Morgado-Valle et al., 2010). Inputs from the NTS or the pons (Kölliker-Fuse and parabrachial nuclei) (Chamberlin, 2004; Rosin et al., 2006; Moreira et al., 2007; Takakura et al., 2007) could also be a source of inhibition.

In contrast to our results in vivo in adult rats, in neonatal rat brainstem en bloc preparations blockade of GABA and glycine receptors synchronizes preBötC and RTN/pFRG activity (Onimaru et al., 1990; Iizuka, 2003; Funke et al., 2008). Notwithstanding the differences between in vitro preparations and whole animals, the different postsynaptic effects of GABA and glycine receptor activation through development may be the result of perinatal changes in $\mathrm{Cl}^{-}$-mediated currents (Ren and Greer,
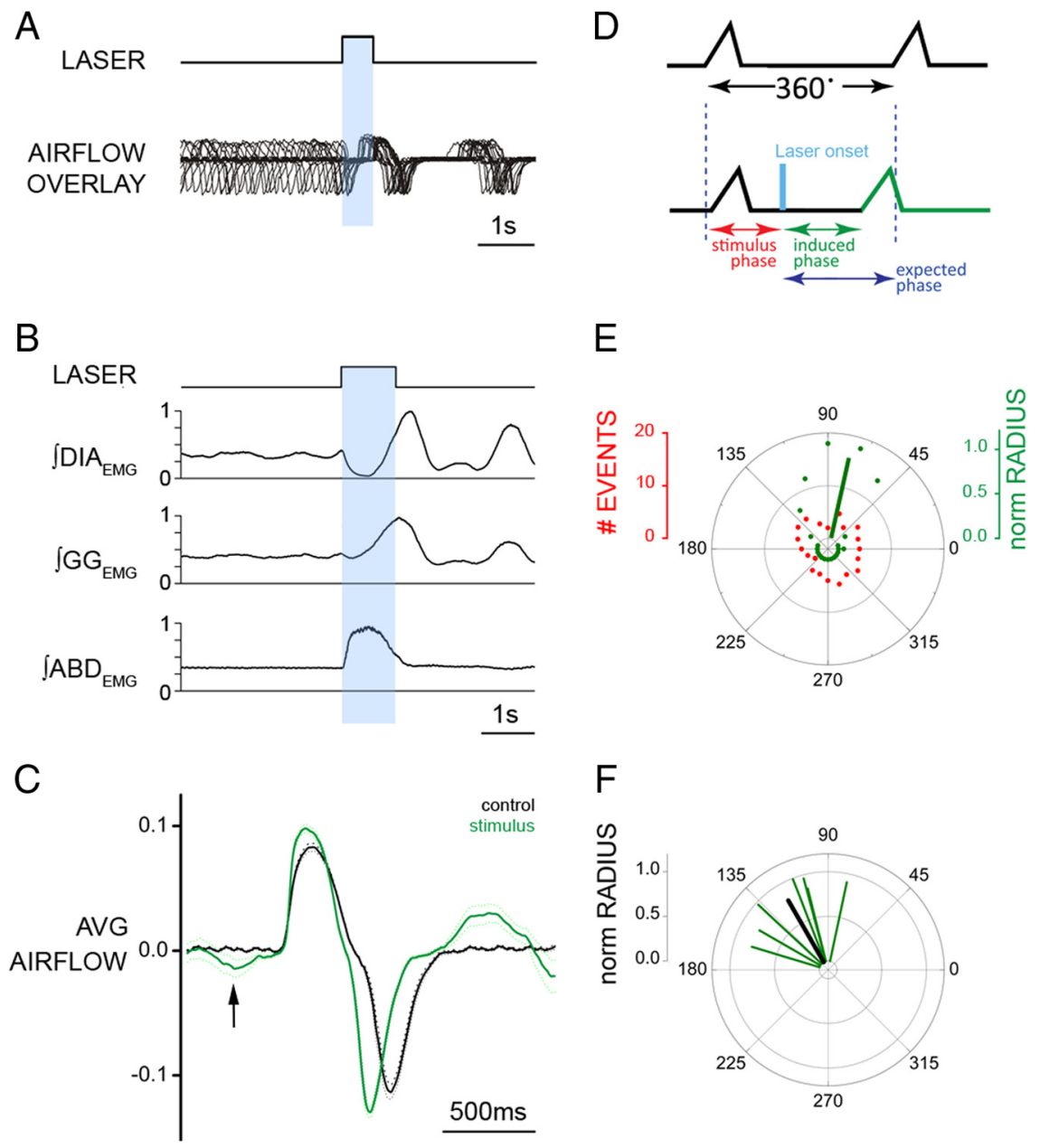

Figure 6. Brief photostimulation of RTN/pFRG neurons resets respiratory cycle. $A$, Superposition of respiratory airflow traces $(n=35)$ during unilateral $500 \mathrm{~ms}$ pulse photostimulation, which resets and aligns the subsequent traces. $\boldsymbol{B}$, Averaged traces for $\int D A_{E M G} \int G_{E M G}$ and $\int A B D_{E M G}$ show reset of EMG on photostimulation ( $1 \mathrm{~s}$ pulse; $n=35$ cycles). Data normalized from 0 to 1 . C, Averaged flow before (black) and after (green) $500 \mathrm{~ms}$ photostimulation ( $n=35$ cycles) aligned to inspiratory onset. The arrow indicates expiratory airflow induced by photostimulation. The dotted line indicates the $95 \%$ confidence interval for the mean. $\boldsymbol{D}$, Phases in reset phase analysis. Top trace, Control respiratory cycle (from 0 to $360^{\circ}$, measured from the onset of one inspiration to the next). Bottom trace, Respiratory cycle during stimulation. Stimulus phase, Onset of photostimulation with respect to the phase of respiration; induced phase, onset of inspiration subsequent to delivery of photostimulation; expected phase, expected onset of the next inspiratory cycle with respect to stimulus onset if photostimulation had no effect $\left(360^{\circ}\right.$ minus stimulus phase). $\boldsymbol{E}$, Distribution of events for stimulus and induced phases during multiple trials of $500 \mathrm{~ms}$ photostimulation delivered during the same experiment $(\boldsymbol{A})$. Although stimuli were distributed randomly across all phases of respiration (red dots), the stimulus-induced phase values (green dots) demonstrated clustering at a tight range of preferred phase angles. The average preferred angle for the induced phase is overlaid as a green vector. The left scale bar indicates radial distance for number of events $(0-20)$ for stimulus or induced phase. The right scale bar indicates normalized radial length for vector $(0-1$, with values close to 1 being indicative of low dispersion of angles and significant phase preferences in polar distributions). $\boldsymbol{F}$, Distribution of preferred phases of stimulusinduced respiration for seven separate experiments plotted as vectors in normalized length units (green) and the calculated grand average (black) across all experiments.

2006). Thus, in the perinatal brainstem, GABA and glycine receptor agonists are likely to excite RTN/pFRG neurons, whereas they would suppress rhythmicity in adulthood.

In adults, expiratory airflow is typically passive at rest, which we hypothesize results, at least in part, from active inhibition of the conditional RTN/pFRG AE oscillator. Whenever metabolic demand increases, such as during exercise or in response to hypercapnea or hypoxia, the RTN/pFRG could be simply disinhibited (and/or excited), allowing for an increase in ventilation via prompt recruitment of $\mathrm{AE}$.

Although in our microinjection experiments we cannot exclude the spreading of drugs medially and ventrally, especially 
A

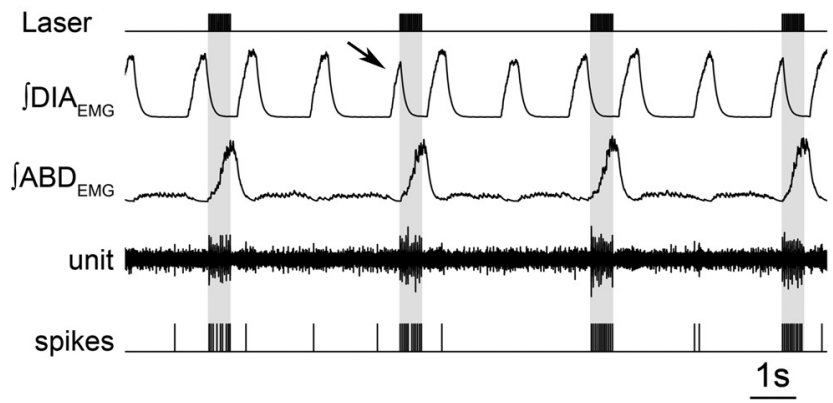

B

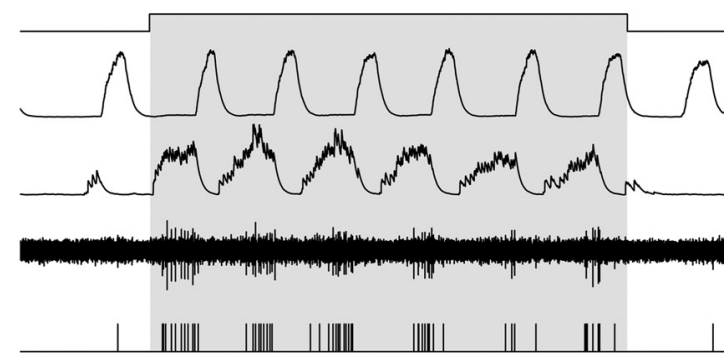

2s

\section{C}

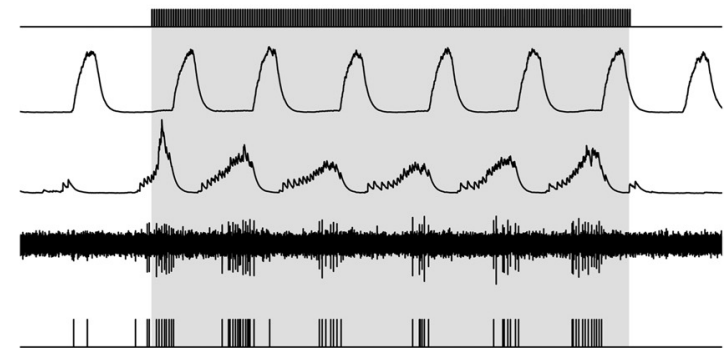

Figure 7. Late expiratory neuron activated by RTN/pFRG photostimulation. $\boldsymbol{A}$, Brief trains $\left(20 \mathrm{~Hz}, 10 \mathrm{~ms}\right.$ ) of photostimulation activate RTN/pFRG neurons and recruits $\mathrm{ABD}_{\mathrm{EMG}}$. Note interruption of inspiratory DIA $A_{E M G}$ (arrow) on photostimulation with sudden recruitment of $A B D_{E M G}$ and expiratory neuron activity. Continuous ( $\left.10 \mathrm{~s}\right)(\boldsymbol{B})$ and brief trains ( $20 \mathrm{~Hz}, 10 \mathrm{~ms}$ for $10 \mathrm{~s})(C)$ promote activation of $A_{B D}$ bursts and neuron fires only during the late expiratory phase.

toward more medial RTN chemosensory neurons (Stornetta et al., 2006; Fortuna et al., 2008; Abbott et al., 2009a), we consistently recorded neurons lateral to the VIIn with late expiratory activity concurrent with ABD muscle recruitment and ultimately AE. As the injected BIC/STRY dissipated and AE waned, these neurons returned to silence. This limitation attributable to injectate spread was addressed in parallel experiments with photoactivation (Nagel et al., 2003; Zhang et al., 2006) of SYN-ChR2-EYFP lentivirus transfected RTN/ pFRG neurons where the population of neurons susceptible to photostimulation was precisely determined by post hoc analysis of the distribution of $\mathrm{EYFP}^{+}$. Our choice of the panneuronal marker synapsin was motivated by a primary test of the hypothesized role of RTN/pFRG unbiased by neuronal phenotype and the fact that, at present, the adult phenotype of the putative AE oscillator is unknown.

Photostimulation of ChR2-transfected RTN/pFRG neurons induced $\mathrm{AE}$, with concurrent phasic activity in $\mathrm{ABD}_{\mathrm{EMG}}, \mathrm{LL}_{\mathrm{EMG}}$, and $\mathrm{GG}_{\mathrm{EMG}}$. Continuous or brief photostimulation could induce prompt $\mathrm{ABD}_{\mathrm{EMG}}$ and $\mathrm{GG}_{\mathrm{EMG}}$ late expiratory activation along with phase-correlated single neuron activity. The firing pattern of the light-activated neurons was independent of the stimulus pat- tern. Brief RTN/pFRG photostimulation reset respiratory rhythm with prompt activation of $\mathrm{AE}$. This timing suggests that recruitment of expiratory activity is attributable to direct stimulation of an $\mathrm{AE}$ oscillator that has a reciprocal inhibitory connection with the inspiratory oscillator and is not secondary to an increased chemoreceptor drive.

Are chemosensitive RTN neurons responsible for generation of AE? At present, there is no evidence of late expiratory activity in chemosensitive RTN/Phox $2 \mathrm{~b}$ neurons of anesthetized adult rats (Mulkey et al., 2004; Guyenet et al., 2005; Stornetta et al., 2006), although neurons with similar properties are present in the same area in an in situ preparation of hypothermic hypercapnic juvenile rats (Abdala et al., 2009). Continuous photostimulation tonically activates RTN Phox $2 \mathrm{~b}^{+}$neurons transfected with ChR2, potentiates inspiratory activity with slow kinetics $\left(t_{1 / 2}=\right.$ $11 \mathrm{~s}$ ), increases overall respiratory drive in behaving rats (Kanbar et al., 2010), but does not appear to reset respiratory rhythm (Abbott et al., 2009a,b; Kanbar et al., 2010). Rather, selective Phox $2 \mathrm{~b}^{+}$neuron photostimulation induces entrainment of Phox $2 \mathrm{~b}^{+}$neuronal activity with phrenic nerve discharge. This is consistent with the proposed role of chemosensitive RTN neurons providing a tonic drive to the respiratory rhythm and pattern generators, but these findings contrast with our results. We consider the following two possibilities: (1) Phox $2 b$ neurons are not rhythmogenic; (2) there are two distinct and partially overlapping regions, a chemosensitive RTN and a rhythmogenic pFRG. During induction of AE with either local application of BIC/STRY or photostimulation, neurons that went from silent to late expiratory modulated were more lateral $(2.3-2.5 \mathrm{~mm}$ from midline) than chemosensitive/Phox $2 \mathrm{~b}^{+}$neurons in adult rats ( 1.6-2.0 mm from midline) (Mulkey et al., 2004; Guyenet et al., 2005; Stornetta et al., 2006). Since the percentage of Phox $2 b$ neurons infected by SYN-ChR2-EYFP was low, and a larger, more lateral neuronal population of non-Phox $2 b$ phenotype was activated by photostimulation, a different neuronal population from the Phox $2 \mathrm{~b}^{+}$chemosensitive neurons ventral to VIIn may well comprise the AE oscillator. The fact that adult Phox $2 \mathrm{~b}^{+}$ RTN neurons (Stornetta et al., 2006; Fortuna et al., 2008; Abbott et al., 2009a) do not exhibit expiratory modulation is either attributable to the experimental approach (e.g., anesthetized rats) or the possibility that through maturation neonatal rhythmogenic neurons lose Phox $2 \mathrm{~b}$ expression, similar to other neuronal populations in the brainstem (Kang et al., 2007).

Extreme hypoxia can induce late expiratory activity in (more caudal) BötC glycinergic neurons in adult anesthetized rats (Fortuna et al., 2008). However, as these neurons were probably not directly affected by BIC/STRY injections, and certainly not transfected by ChR2, they are unlikely to be the first link in the chain inducing AE. Although we cannot rule out the possibility that our stimuli triggered a conditional BötC expiratory oscillator, the existence of RTN/pFRG neurons that became late expiratorymodulated when AE was induced [as well as from the fact that they are rhythmic in vitro (Onimaru et al., 2008; Thoby-Brisson et al., 2009)] offers a more parsimonious interpretation consistent with our hypothesis: the RTN/pFRG is a conditional expiratory oscillator.

Transient photostimulation produced consistent changes in respiratory timing, with sudden onset of expiratory muscle activity that effectively delayed the onset of inspiration. The timing of this response before inspiration onset suggests that it is not secondary to the stimulation of the inspiratory oscillator (presumably the preBötC) (Feldman and Del Negro, 2006) or the result of an additional tonic drive to the preBötC, as suggested for RTN 
Phox 2b neuron photostimulation (Abbott et al., 2009a). The most efficient respiratory cycle phase to induce $\mathrm{AE}$ and a prompt reset of inspiration was at the end of expiration (when AE would spontaneously occur, if present) or at the beginning of inspiration.

AE but not inspiration is lost when the RTN/pFRG is removed after transverse medullary section just rostral to the preBötC and BötC in anesthetized juvenile rats (Janczewski and Feldman, 2006). Here, we show that AE is induced by activation of RTN/ pFRG by either disinhibition or photostimulation in anesthetized adult rats. This is consistent with the RTN/pFRG playing a causal role in the generation of $\mathrm{AE}$ at all stages of postnatal development. Furthermore, the observation that tonic/silent RTN/pFRG neurons become rhythmic with a late expiratory firing pattern is consistent with our hypothesis that this region contains a conditional oscillator that drives expiratory motor activity, even in adulthood. Given that expiration is only present in certain states suggests that this conditional oscillator is turned on in situations when active expiration is necessary to efficiently increase ventilation, as occurs during exercise.

\section{References}

Abbott SB, Stornetta RL, Fortuna MG, Depuy SD, West GH, Harris TE, Guyenet PG (2009a) Photostimulation of retrotrapezoid nucleus phox $2 \mathrm{~b}$-expressing neurons in vivo produces long-lasting activation of breathing in rats. J Neurosci 29:5806-5819.

Abbott SB, Stornetta RL, Socolovsky CS, West GH, Guyenet PG (2009b) Photostimulation of channelrhodopsin-2 expressing ventrolateral medullary neurons increases sympathetic nerve activity and blood pressure in rats. J Physiol 587:5613-5631.

Abdala AP, Rybak IA, Smith JC, Paton JF (2009) Abdominal expiratory activity in the rat brainstem-spinal cord in situ: patterns, origins and implications for respiratory rhythm generation. J Physiol 587:3539-3559.

Abraham KA, Feingold H, Fuller DD, Jenkins M, Mateika JH, Fregosi RF (2002) Respiratory-related activation of human abdominal muscles during exercise. J Physiol 541:653-663.

Adamantidis AR, Zhang F, Aravanis AM, Deisseroth K, de Lecea L (2007) Neural substrates of awakening probed with optogenetic control of hypocretin neurons. Nature 450:420-424.

Chamberlin NL (2004) Functional organization of the parabrachial complex and intertrigeminal region in the control of breathing. Respir Physiol Neurobiol 143:115-125.

Connelly CA, Ellenberger HH, Feldman JL (1990) Respiratory activity in retrotrapezoid nucleus in cat. Am J Physiol 258:L33-L44.

Cream C, Li A, Nattie E (2002) The retrotrapezoid nucleus (RTN): local cytoarchitecture and afferent connections. Respir Physiol Neurobiol 130:121-137.

de Almeida AT, Kirkwood PA (2010) Multiple phases of excitation and inhibition in central respiratory drive potentials of thoracic motoneurones in the rat. J Physiol 588:2731-2744.

de Almeida AT, Al-Izki S, Denton ME, Kirkwood PA (2010) Patterns of expiratory and inspiratory activation for thoracic motoneurones in the anaesthetized and the decerebrate rat. J Physiol 588:2707-2729.

Feldman JL, Del Negro CA (2006) Looking for inspiration: new perspectives on respiratory rhythm. Nat Rev Neurosci 7:232-242.

Feldman JL, Smith JC (1989) Cellular mechanisms underlying modulation of breathing pattern in mammals. Ann N Y Acad Sci 563:114-130.

Feldman JL, Kam K, Janczewski WA (2009) Practice makes perfect, even for breathing. Nat Neurosci 12:961-963.

Fortuna MG, West GH, Stornetta RL, Guyenet PG (2008) Botzinger expiratory-augmenting neurons and the parafacial respiratory group. J Neurosci 28:2506-2515.

Funke F, Müller M, Dutschmann M (2008) Reconfiguration of respiratoryrelated population activity in a rostrally tilted transversal slice preparation following blockade of inhibitory neurotransmission in neonatal rats. Pflugers Arch 457:185-195.

Gradinaru V, Thompson KR, Zhang F, Mogri M, Kay K, Schneider MB, Deisseroth K (2007) Targeting and readout strategies for fast optical neural control in vitro and in vivo. J Neurosci 27:14231-14238.
Gray PA, Rekling JC, Bocchiaro CM, Feldman JL (1999) Modulation of respiratory frequency by peptidergic input to rhythmogenic neurons in the preBotzinger complex. Science 286:1566-1568.

Gray PA, Janczewski WA, Mellen N, McCrimmon DR, Feldman JL (2001) Normal breathing requires preBotzinger complex neurokinin-1 receptorexpressing neurons. Nat Neurosci 4:927-930.

Guyenet PG, Mulkey DK, Stornetta RL, Bayliss DA (2005) Regulation of ventral surface chemoreceptors by the central respiratory pattern generator. J Neurosci 25:8938-8947.

Guyenet PG, Stornetta RL, Bayliss DA (2008) Retrotrapezoid nucleus and central chemoreception. J Physiol 586:2043-2048.

lizuka M (2003) $\mathrm{GABA}_{\mathrm{A}}$ and glycine receptors in regulation of intercostal and abdominal expiratory activity in vitro in neonatal rat. J Physiol 551:617-633.

Iizuka M, Fregosi RF (2007) Influence of hypercapnic acidosis and hypoxia on abdominal expiratory nerve activity in the rat. Respir Physiol Neurobiol 157:196-205.

Iscoe S (1998) Control of abdominal muscles. Prog Neurobiol 56:433-506.

Janczewski WA, Feldman JL (2006) Distinct rhythm generators for inspiration and expiration in the juvenile rat. J Physiol 570:407-420.

Janczewski WA, Onimaru H, Homma I, Feldman JL (2002) Opioidresistant respiratory pathway from the preinspiratory neurones to abdominal muscles: in vivo and in vitro study in the newborn rat. J Physiol 545:1017-1026.

Kanbar R, Stornetta RL, Cash DR, Lewis SJ, Guyenet PG (2010) Photostimulation of Phox $2 \mathrm{~b}$ medullary neurons activates cardiorespiratory function in conscious rats. Am J Respir Crit Care Med 182:1184-1194.

Kang BJ, Chang DA, Mackay DD, West GH, Moreira TS, Takakura AC, Gwilt JM, Guyenet PG, Stornetta RL (2007) Central nervous system distribution of the transcription factor Phox $2 b$ in the adult rat. J Comp Neurol 503:627-641.

Marina N, Abdala AP, Trapp S, Li A, Nattie EE, Hewinson J, Smith JC, Paton JF, Gourine AV (2010) Essential role of Phox2b-expressing ventrolateral brainstem neurons in the chemosensory control of inspiration and expiration. J Neurosci 30:12466-12473.

Mellen NM, Janczewski WA, Bocchiaro CM, Feldman JL (2003) Opioidinduced quantal slowing reveals dual networks for respiratory rhythm generation. Neuron 37:821-826.

Moreira TS, Takakura AC, Colombari E, West GH, Guyenet PG (2007) Inhibitory input from slowly adapting lung stretch receptors to retrotrapezoid nucleus chemoreceptors. J Physiol 580:285-300.

Morgado-Valle C, Baca SM, Feldman JL (2010) Glycinergic pacemaker neurons in preBotzinger complex of neonatal mouse. J Neurosci 30:3634-3639.

Mulkey DK, Stornetta RL, Weston MC, Simmons JR, Parker A, Bayliss DA, Guyenet PG (2004) Respiratory control by ventral surface chemoreceptor neurons in rats. Nat Neurosci 7:1360-1369.

Nagel G, Szellas T, Huhn W, Kateriya S, Adeishvili N, Berthold P, Ollig D, Hegemann P, Bamberg E (2003) Channelrhodopsin-2, a directly lightgated cation-selective membrane channel. Proc Natl Acad Sci U S A 100:13940-13945.

Onimaru H, Homma I (2003) A novel functional neuron group for respiratory rhythm generation in the ventral medulla. J Neurosci 23:1478-1486.

Onimaru H, Arata A, Homma I (1990) Inhibitory synaptic inputs to the respiratory rhythm generator in the medulla isolated from newborn rats. Pflugers Arch 417:425-432.

Onimaru H, Arata A, Homma I (1995) Intrinsic burst generation of preinspiratory neurons in the medulla of brainstem-spinal cord preparations isolated from newborn rats. Exp Brain Res 106:57-68.

Onimaru H, Kumagawa Y, Homma I (2006) Respiration-related rhythmic activity in the rostral medulla of newborn rats. J Neurophysiol 96:55-61.

Onimaru H, Ikeda K, Kawakami $\mathrm{K}$ (2008) $\mathrm{CO}_{2}$-sensitive preinspiratory neurons of the parafacial respiratory group express Phox $2 \mathrm{~b}$ in the neonatal rat. J Neurosci 28:12845-12850.

Pagliardini S, Tan, W, Janczewski WA, Feldman JL (2009a) Excitation and disinhibition of RTN/pFRG neurons induce active expiration in adult rats. Paper presented at XIth Annual Oxford Conference on Modeling and Control of Breathing: New Frontiers in Respiratory Control. Nara, Japan, July.

Pagliardini S, Tan W, Janczewski WA, Feldman JL (2009b) Excitation of parafacial neurons induces active expiration in adult rats. Soc Neurosci Abstr 35:89.6 
Paxinos G, Watson C (1998) The rat brain in stereotaxic coordinates, Ed 4. New York: Academic.

Pearce RA, Stornetta RL, Guyenet PG (1989) Retrotrapezoid nucleus in the rat. Neurosci Lett 101:138-142.

Ren J, Greer JJ (2006) Modulation of respiratory rhythmogenesis by chloride-mediated conductances during the perinatal period. J Neurosci 26:3721-3730.

Rosin DL, Chang DA, Guyenet PG (2006) Afferent and efferent connections of the rat retrotrapezoid nucleus. J Comp Neurol 499:64-89.

Sears TA, Berger AJ, Phillipson EA (1982) Reciprocal tonic activation of inspiratory and expiratory motoneurones by chemical drives. Nature 299:728-730.

Smith JC, Morrison DE, Ellenberger HH, Otto MR, Feldman JL (1989) Brainstem projections to the major respiratory neuron populations in the medulla of the cat. J Comp Neurol 281:69-96.

Smith JC, Ellenberger HH, Ballanyi K, Richter DW, Feldman JL (1991) PreBotzinger complex: a brainstem region that may generate respiratory rhythm in mammals. Science 254:726-729.

Stornetta RL, Moreira TS, Takakura AC, Kang BJ, Chang DA, West GH, Brunet JF, Mulkey DK, Bayliss DA, Guyenet PG (2006) Expression of
Phox $2 \mathrm{~b}$ by brainstem neurons involved in chemosensory integration in the adult rat. J Neurosci 26:10305-10314.

Takakura AC, Moreira TS, West GH, Gwilt JM, Colombari E, Stornetta RL, Guyenet PG (2007) GABAergic pump cells of solitary tract nucleus innervate retrotrapezoid nucleus chemoreceptors. J Neurophysiol 98: $374-381$.

Tan W, Janczewski WA, Yang P, Shao XM, Callaway EM, Feldman JL (2008) Silencing preBotzinger complex somatostatin-expressing neurons induces persistent apnea in awake rat. Nat Neurosci 11: $538-540$

Thoby-Brisson M, Karlén M, Wu N, Charnay P, Champagnat J, Fortin G (2009) Genetic identification of an embryonic parafacial oscillator coupling to the preBotzinger complex. Nat Neurosci 12:1028-1035.

Zar JH (1996) Biostatistical analysis, Ed 3. Upper Saddle River, NJ: Prentice Hall.

Zhang F, Wang LP, Boyden ES, Deisseroth K (2006) Channelrhodopsin-2 and optical control of excitable cells. Nat Methods 3:785-792.

Zhang F, Wang LP, Brauner M, Liewald JF, Kay K, Watzke N, Wood PG, Bamberg E, Nagel G, Gottschalk A, Deisseroth K (2007) Multimodal fast optical interrogation of neural circuitry. Nature 446:633-639. 